\title{
Graph equivariant cohomological rigidity for GKM graphs
}

\author{
By Matthias Franz ${ }^{*}$ and Hitoshi YAmanaKA*) \\ (Communicated by Masaki KashiwarA, M.J.A., Nov. 12, 2019)
}

\begin{abstract}
We formulate the notion of an isomorphism of GKM graphs. We then show that two GKM graphs have isomorphic graph equivariant cohomology algebras if and only if the graphs are isomorphic.
\end{abstract}

Key words: GKM graph; graph equivariant cohomology; equivariant cohomological rigidity.

1. Introduction. In [3, Thm. 7.2], GoreskyKottwitz-MacPherson found a remarkable combinatorial description of the equivariant cohomology, with complex coefficients, of a complex projective variety with an algebraic torus action having only finitely many 1-dimensional orbits. Subsequently, Guillemin-Zara $[4, \S 1.6]$ generalized their work to a wide class of closed $T$-manifolds, where $T$ is a compact torus. Nowadays, a manifold appearing in this class is called a GKM manifold.

A GKM manifold $X$ determines an edgelabeled graph $\mathcal{G}_{X}$ that encodes the structure of the equivariant 1-skeleton of $X$ and the weights of the tangential real representations. Using these data one can define the graph equivariant cohomology $H_{T}^{*}\left(\mathcal{G}_{X}\right)$ in a purely combinatorial way. It is a sub graded $H^{*}(B T)$-algebra of the algebra of functions from the fixed point set $X^{T}$ to $H^{*}(B T)$.

The theorem of Goresky-Kottwitz-MacPherson and of Guillemin-Zara mentioned above asserts that if the equivariant cohomology of a GKM manifold $X$ is free over $H^{*}(B T)$, then $H_{T}^{*}(X)$ is canonically isomorphic to $H_{T}^{*}\left(\mathcal{G}_{X}\right)$ as an $H^{*}(B T)$ algebra. This holds for complex coefficients, and also for integral coefficients provided that all isotropy groups in $X$ are connected, see Remark 2.4 below.

The work of Guillemin-Zara has another important aspect: they established an axiomatic formulation of the framework above by introducing the notion of an abstract GKM graph $\mathcal{G}$ and its

2010 Mathematics Subject Classification. Primary 55N91; Secondary 57S15.

*) Department of Mathematics, University of Western Ontario, London, Ont. N6A B57, Canada.

**) Osaka City University Advanced Mathematical Institute, 3-3-138, Sugimoto, Sumiyoshi-ku, Osaka 558-8585, Japan. graph equivariant cohomology $H_{T}^{*}(\mathcal{G})$ with integral coefficients, which is a graded algebra over the integral cohomology $H^{*}(B T)$.

Toric manifolds are important examples of GKM manifolds. In [6, Thm. 1.1] Masuda proved that the equivariant isomorphism type of a toric manifold, considered as a complex algebraic variety with an algebraic torus action, is completely determined by its torus equivariant cohomology algebra with integral coefficients. This work has led to a classification problem in toric topology which is nowadays called the cohomological rigidity problem. The aim of the present note is to generalize Masuda's result to arbitrary GKM graphs.

Let $\mathcal{G}$ and $\mathcal{G}^{\prime}$ be two abstract GKM graphs defined for the same torus $T$ (see Definition 2.3). We denote by $H_{T}^{*}(\mathcal{G})$ and $H_{T}^{*}\left(\mathcal{G}^{\prime}\right)$ the corresponding graph equivariant cohomology of $\mathcal{G}$ and $\mathcal{G}^{\prime}$, respectively (see Definition 2.5). In Definition 2.6 we will introduce the notion of an isomorphism $\varphi: \mathcal{G}^{\prime} \rightarrow \mathcal{G}$. Our main theorem is the following

Theorem 1.1. $H_{T}^{*}(\mathcal{G})$ and $H_{T}^{*}\left(\mathcal{G}^{\prime}\right)$ are isomorphic as $H^{*}(B T)$-algebras if and only if $\mathcal{G}$ and $\mathcal{G}^{\prime}$ are isomorphic as GKM graphs.

For a toric manifold $X$, the GKM graph $\mathcal{G}_{X}$ and the fan $\Sigma_{X}$ are essentially the same object. Since isotropy groups in toric varieties are connected, Theorem 1.1 generalizes Masuda's equivariant rigidity theorem to abstract GKM graphs.

Throughout this note, we fix a compact torus $T$ of rank $r$ as well as positive integers $n$ and $n^{\prime}$. Note that $H^{*}(B T)$ can be regarded as the polynomial ring $\mathbf{Z}\left[x_{1}, \ldots, x_{r}\right]$ with the grading $\operatorname{deg} x_{i}=2$. In particular, it is a unique factorization domain. For two polynomials $P, Q \in H^{*}(B T)$, we write $P \mid Q$ if $Q=R P$ for some $R \in H^{*}(B T)$. We denote by $|S|$ the number of elements of a finite set $S$. 
2. Graph equivariant cohomology. In this section we recall the notion of an abstract GKM graph and its graph equivariant cohomology. The original paper is [4]. We also introduce the notion of an isomorphism of GKM graphs.

Let $\mathcal{G}$ be a finite $n$-valent undirected graph (multi-edges are allowed, but loops are not) with vertex set $\mathcal{V}$. We denote by $\mathcal{E}$ the set of directed edges of $\mathcal{G}$. (Note that $\mathcal{E}$ is not the set of edges of $\mathcal{G}$; the cardinality of $\mathcal{E}$ is twice that of the edge set.) For each $e \in \mathcal{E}$, we denote by $\bar{e}$ the directed edge obtained by reversing the direction of $e$. Let $i(e)$ and $t(e)$ be the initial and terminal point of a directed edge $e$, respectively. We also use the following notation for vertices $p$ and $q$ :

$$
\begin{gathered}
\mathcal{E}_{p}:=\{e \in \mathcal{E} \mid i(e)=p\}, \\
\mathcal{E}_{p q}:=\{e \in \mathcal{E} \mid i(e)=p, t(e)=q\} .
\end{gathered}
$$

Definition 2.1. A map $\alpha: \mathcal{E} \rightarrow H^{2}(B T)$ is called an axial function on $\mathcal{G}$ if it satisfies the following three conditions for all $e, e^{\prime} \in \mathcal{E}$ :

(i) $\alpha(\bar{e})= \pm \alpha(e)$.

(ii) (GKM condition) $\alpha(e)$ and $\alpha\left(e^{\prime}\right)$ are linearly independent over $\mathbf{Z}$ if $e \neq e^{\prime}$ and $i(e)=i\left(e^{\prime}\right)$.

(iii) (Primitivity) The greatest common divisor of the coefficients of $\alpha(e)$ is 1 .

Definition 2.2. Let $\alpha$ be an axial function on $\mathcal{G}$. A parallel transport of $(\mathcal{G}, \alpha)$ is a family $\mathcal{P}=$ $\left\{\mathcal{P}_{e}\right\}_{e \in \mathcal{E}}$ of bijections $\mathcal{P}_{e}: \mathcal{E}_{i(e)} \rightarrow \mathcal{E}_{t(e)}$ satisfying the following conditions for all $e \in \mathcal{E}$ and all $e^{\prime} \in \mathcal{E}_{i(e)}$ :

(i) $\mathcal{P}_{\bar{e}}=\mathcal{P}_{e}^{-1}$.

(ii) $\mathcal{P}_{e}(e)=\bar{e}$.

(iii) $\alpha\left(\mathcal{P}_{e}\left(e^{\prime}\right)\right)-\alpha\left(e^{\prime}\right) \in \mathbf{Z} \alpha(e)$.

Definition 2.3. An abstract GKM graph (or simply $G K M$ graph) of type $(r, n)$ is a pair $(\mathcal{G}, \alpha)$ having at least one parallel transport.

Remark 2.4. The above notation and terminology are somewhat different from the usual ones. Let us explain the differences.

(i) Condition (i) in Definition 2.1 is weaker than the usual requirement $\alpha(\bar{e})=-\alpha(e)$. Our definition is motivated by the notion of a torus graph introduced in $[5, \S 3]$, and is more natural from the point of view of real manifolds.

(ii) Condition (iii) in Definition 2.1 is related to our choice of integers as the coefficient ring for graph equivariant cohomology. For complex coefficients, it would hold trivially. If we want the theorem of Goresky-Kottwitz-MacPherson and of Guillemin-Zara to hold in the case of integral coefficients, we have to put further assumptions on the GKM manifold $X$. One possible condition is the connectedness of the stabilizer group $T_{x}$ for any $x \in X$, see [1, Thm. 1.1] or [2, Thm. 2.1]. The primitivity condition reflects the connectedness of the stabilizer groups for the equivariant 1-skeleton in a purely algebraic fashion. Many known GKM manifolds with effective torus action satisfy it.

(iii) The family $\mathcal{P}$ is usually called a connection on $(\mathcal{G}, \alpha)$. As well-explained in [4], this terminology owes its origin to the "fiber bundle" picture for GKM graphs, see $[4, \S 1.7]$. However, it seems more appropriate to call it a parallel transport since each bijection $\mathcal{P}_{e}$ corresponds to an identification of fibers.

Definition 2.5. The graph equivariant cohomology of a GKM graph $(\mathcal{G}, \alpha)$ is defined to be

$$
\begin{aligned}
H_{T}^{*}(\mathcal{G})= & \left\{f: \mathcal{V} \rightarrow H^{*}(B T) \mid\right. \\
& \alpha(e) \mid(f(i(e))-f(t(e)))(e \in \mathcal{E})\} ;
\end{aligned}
$$

it is a sub graded $H^{*}(B T)$-algebra of the algebra of all functions $\mathcal{V} \rightarrow H^{*}(B T)$. We denote by $H_{T}^{2 i}(\mathcal{G})$ its degree $2 i$ component where $f \in H_{T}^{*}(\mathcal{G})$ is of degree $2 i$ if $f(p)$ is so for any $p \in \mathcal{V}$.

We now introduce the notion of an isomorphism of GKM graphs. For $p \neq q \in \mathcal{V}$, we set

$$
P_{p q}:=\prod_{e \in \mathcal{E}_{p q}} \alpha(e) .
$$

Note that $P_{p q} \neq 1$ if and only if $p$ and $q$ are adjacent. Let $\left(\mathcal{G}^{\prime}, \alpha^{\prime}\right)$ be a GKM graph of type $\left(r, n^{\prime}\right)$.

Definition 2.6. An isomorphism $\varphi: \mathcal{G}^{\prime} \rightarrow \mathcal{G}$ of GKM graphs is a bijection $\varphi_{\mathcal{V}}: \mathcal{V}^{\prime} \rightarrow \mathcal{V}$ such that for all $p^{\prime}, q^{\prime} \in \mathcal{V}^{\prime}$ one has $P_{\varphi_{\mathcal{V}}\left(p^{\prime}\right) \varphi_{\mathcal{V}}\left(q^{\prime}\right)}= \pm P_{p^{\prime} q^{\prime}}$.

This implies that $p^{\prime}$ and $q^{\prime}$ are adjacent if and only if $\varphi_{\mathcal{V}}\left(p^{\prime}\right)$ and $\varphi_{\mathcal{V}}\left(q^{\prime}\right)$ are so. Two GKM graphs $\mathcal{G}$, $\mathcal{G}^{\prime}$ are said to be isomorphic if there exists an isomorphism $\mathcal{G}^{\prime} \rightarrow \mathcal{G}$ of GKM graphs.

In light of the primitivity condition, the criterion stated in Definition 2.6 can be paraphrased as follows:

For any two vertices $p^{\prime}, q^{\prime}$ of $\mathcal{G}^{\prime}$ there exists a bijection $\varphi_{\mathcal{E}}: \mathcal{E}_{\varphi_{\nu}\left(p^{\prime}\right) \varphi_{\nu}\left(q^{\prime}\right)} \rightarrow \mathcal{E}_{p^{\prime} q^{\prime}}$ such that $\alpha^{\prime}\left(\varphi_{\mathcal{E}}(e)\right)= \pm \alpha(e)$. Here we are using that each weight $\alpha(e)$ is a prime element of the UFD $H^{*}(B T)$ by the primitivity condition.

Remark 2.7. The preceding reformulation 
implies that any isomorphism $\varphi: \mathcal{G}^{\prime} \rightarrow \mathcal{G}$ induces a graded $H^{*}(B T)$-algebra isomorphism $\varphi^{*}: H_{T}^{*}(\mathcal{G}) \rightarrow$ $H_{T}^{*}\left(\mathcal{G}^{\prime}\right)$ defined by $\left(\varphi^{*}(f)\right)\left(p^{\prime}\right):=f\left(\varphi_{\mathcal{V}}\left(p^{\prime}\right)\right)$. The assignment is functorial in the sense that $\operatorname{id}_{\mathcal{G}}^{*}=$ $\operatorname{id}_{H_{T}^{*}(\mathcal{G})}$ and $(\psi \circ \varphi)^{*}=\varphi^{*} \circ \psi^{*}$ for isomorphisms $\varphi: \mathcal{G}^{\prime \prime} \rightarrow \mathcal{G}^{\prime}, \psi: \mathcal{G}^{\prime} \rightarrow \mathcal{G}$.

3. Equivariant Thom classes. Following Guillemin-Zara $[4, \S 2.3]$, we introduce the equivariant Thom class corresponding to a vertex of a GKM graph $(\mathcal{G}, \alpha)$.

Definition 3.1. For any $p \in \mathcal{V}$, we define a map $\tau_{p}: \mathcal{V} \rightarrow H^{*}(B T)$ by

$$
\tau_{p}(q):= \begin{cases}\prod_{e \in \mathcal{E}_{p}} \alpha(e) & \text { if } q=p, \\ 0 & \text { if } q \neq p .\end{cases}
$$

The map $\tau_{p}$ is called the equivariant Thom class associated with $p$; it is an element of $H_{T}^{2 n}(\mathcal{G})$.

Remark 3.2. Assume that $\mathcal{G}$ is the GKM graph of a toric manifold $X$ given by a complete fan $\Sigma$. Then each $p \in X^{T}=\mathcal{V}$ is the transverse intersection of $n$ invariant divisors $X_{i_{1}}, \ldots, X_{i_{n}}$. Our $\tau_{p}$ corresponds to the Thom class $\tau_{i_{1}} \cdots \tau_{i_{n}} \in H_{T}^{2 n}(X)$ of $p$ in $X$. The Thom class $\tau_{i}$ of $X_{i}(c f .[6$, p. 2007]) is represented by the function $\xi_{i}: \mathcal{V} \rightarrow H^{*}(B T)$ given by

$$
\xi_{i}(p)= \begin{cases}\alpha(e) & \text { if } p \in X_{i}, \\ 0 & \text { otherwise }\end{cases}
$$

where $e \in \mathcal{E}$ is the unique edge from $p$ to a $q \notin X_{i}$, see $[7, \S 6.2]$.

Lemma 3.3. Let $F$ be a subset of $H_{T}^{*}(\mathcal{G}) \backslash\{0\}$ such that $f g=0$ for all distinct $f, g \in F$. Then $|F| \leq|\mathcal{V}|$. Equality holds if and only if each $f$ is supported at a single vertex and each vertex occurs as the support of some $f \in F$.

Proof. Let $\mathcal{V}_{f}:=\{p \in \mathcal{V} \mid f(p) \neq 0\}$ be the support of $f \in \mathcal{V}$. The assumptions imply $\mathcal{V}_{f} \neq \emptyset$ and $\mathcal{V}_{f} \cap \mathcal{V}_{g}=\emptyset$ for distinct $f, g$. Thus the inequality holds. The equality is attained if and only if each $\mathcal{V}_{f}$ is a singleton and $\mathcal{V}$ is the union of the $\mathcal{V}_{f}$ 's.

The following result gives a ring-theoretic characterization of the set of equivariant Thom classes $\left\{\tau_{p}\right\}_{p \in \mathcal{V}}$, up to sign.

Proposition 3.4. The set $F=\left\{\tau_{p} \mid p \in \mathcal{V}\right\}$ is a maximal collection of elements as in Lemma 3.3, and each other maximal collection is obtained by scaling each equivariant Thom class by some element in $H^{*}(B T)$. These properties characterize
F up to signs.

Proof. This follows from Lemma 3.3 and the definition of $H_{T}^{*}(\mathcal{G})$.

4. Key lemma. Throughout this section, we fix vertices $p \neq q$ of $\mathcal{G}$. We then introduce the following polynomials:

$$
P:=\prod_{e \in \mathcal{E}_{p} \backslash \mathcal{E}_{p q}} \alpha(e), \quad Q:=\prod_{e \in \mathcal{E}_{q} \backslash \mathcal{E}_{q p}} \alpha(e) .
$$

For any $e \in \mathcal{E}_{p q}$ we set

$$
c(e):=\left|\left\{e^{\prime} \in \mathcal{E}_{p q} \mid e^{\prime} \neq e, \alpha\left(\overline{e^{\prime}}\right)=-\alpha\left(e^{\prime}\right)\right\}\right| .
$$

The following is the key lemma in our proof. For its proof the existence of a parallel transport on $\mathcal{G}$ is essential.

Lemma 4.1. For any $e \in \mathcal{E}_{p q}$, the polynomial $P-(-1)^{c(e)} Q$ is divisible by $\alpha(e)$.

Proof. Let $\mathcal{P}$ be a parallel transport over $\mathcal{G}$. By condition (iii) in Definition 2.2, there exist integers $\left\{d_{e, e^{\prime}}\right\}_{e^{\prime} \in \mathcal{E}_{p}}$ satisfying

$$
\alpha\left(\mathcal{P}_{e}\left(e^{\prime}\right)\right)-\alpha\left(e^{\prime}\right)=d_{e, e^{\prime}} \alpha(e)
$$

for any $e^{\prime} \in \mathcal{E}_{p}$. Using these relations, we have

$$
\begin{aligned}
& P \cdot \prod_{\substack{e^{\prime} \in \mathcal{E}_{p q} \\
e^{\prime} \neq e}} \alpha\left(e^{\prime}\right)=\prod_{\substack{e^{\prime} \in \mathcal{E}_{p} \\
e^{\prime} \neq e}} \alpha\left(e^{\prime}\right) \\
&= \prod_{\substack{e^{\prime} \in \mathcal{E}_{p} \\
e^{\prime} \neq e}}\left(\alpha\left(\mathcal{P}_{e}\left(e^{\prime}\right)\right)-d_{e, e^{\prime}} \alpha(e)\right) \\
& \equiv \prod_{\substack{e^{\prime} \in \mathcal{E}_{p} \\
e^{\prime} \neq e}} \alpha\left(\mathcal{P}_{e}\left(e^{\prime}\right)\right)=\prod_{\substack{e^{\prime \prime} \in \mathcal{E}_{q} \\
e^{\prime \prime} \neq \bar{e}}} \alpha\left(e^{\prime \prime}\right) \\
&=Q \cdot \prod_{\substack{e^{\prime \prime} \in \mathcal{E}_{q p} \\
e^{\prime \prime} \neq \bar{e}}} \alpha\left(e^{\prime \prime}\right)=Q \cdot(-1)^{c(e)} \prod_{\substack{e^{\prime} \in \mathcal{E}_{p q} \\
e^{\prime} \neq e}} \alpha\left(e^{\prime}\right) ;
\end{aligned}
$$

here "三” means equality modulo $\alpha(e)$ (in other words, equality in the quotient ring $H^{*}(B T) /$ $\langle\alpha(e)\rangle)$.

Thus

$$
\left(P-(-1)^{c(e)} Q\right) \prod_{\substack{e^{\prime} \in \mathcal{E}_{p q} \\ e^{\prime} \neq e}} \alpha\left(e^{\prime}\right)
$$

is divisible by $\alpha(e)$ as $\alpha(e)$ is a prime element in $H^{*}(B T)$. Since $\alpha\left(e^{\prime}\right)\left(e^{\prime} \in \mathcal{E}_{p q}, e^{\prime} \neq e\right)$ and $\alpha(e)$ are coprime by the GKM condition, the proof is now complete.

We set

$$
\begin{aligned}
& E:=\left\{e \in \mathcal{E}_{p q} \mid c(e) \text { is even }\right\}, \\
& O:=\left\{e \in \mathcal{E}_{p q} \mid c(e) \text { is odd }\right\} .
\end{aligned}
$$


Notice that $\mathcal{E}_{p q}$ is the disjoint union of $E$ and $O$. Lemma 4.1 immediately implies the following

Corollary 4.2. The polynomials $P-Q$ and $P+Q$ are divisible by $\prod_{e \in E} \alpha(e)$ and $\prod_{e \in O} \alpha(e)$, respectively.

5. Proof of the main theorem. Now we are in the position to prove our main theorem:

Theorem 5.1. $H_{T}^{*}(\mathcal{G})$ and $H_{T}^{*}\left(\mathcal{G}^{\prime}\right)$ are isomorphic as $H^{*}(B T)$-algebras if and only if $\mathcal{G}$ and $\mathcal{G}^{\prime}$ are isomorphic as GKM graphs.

Proof. The "if" part follows from Remark 2.7.

We have seen in Proposition 3.4 that from the $H^{*}(B T)$-algebra $H_{T}^{*}(\mathcal{G})$ we can recover the equivariant Thom classes, up to sign, and in particular the vertex set. We show that one can also recover the polynomials $P_{p q}$. This will prove the claim.

Let $p, q$ be distinct vertices. We define a map $f: \mathcal{V} \rightarrow H^{*}(B T)$ by

$$
f(v):= \begin{cases}P^{2} & \text { if } v=p \\ Q^{2} & \text { if } v=q, \\ 0 & \text { otherwise }\end{cases}
$$

We first check that $f$ is in $H_{T}^{*}(\mathcal{G})$.

By Corollary 4.2, $f(p)-f(q)=(P+Q)(P-Q)$ is divisible by

$$
\left(\prod_{e \in E} \alpha(e)\right) \cdot\left(\prod_{e \in O} \alpha(e)\right)=P_{p q} .
$$

Together with the definition of $P$ and $Q$, this implies that $f$ is an element of $H_{T}^{*}(\mathcal{G})$.

We note that the identity $P_{p q}^{2} f=\tau_{p}^{2}+\tau_{q}^{2}$ holds. In the rest of the proof, we show that the degree of $P_{p q}$ and this identity characterize $\pm P_{p q}$.

Assume that an element $R \in H^{*}(B T)$ satisfies the identity $R^{2} g=\tau_{p}^{2}+\tau_{q}^{2}$ for some $g \in H_{T}^{*}(\mathcal{G})$. Then $g(v)=0$ for all $v \in \mathcal{V} \backslash\{p, q\}$, and $g(p)$ is divisible by $P$. Since $g(p)=\left(\tau_{p}(p) / R\right)^{2}$ and $P$ is square-free, we see that $g(p)$ is even divisible by $P^{2}$. Because $P_{p q}^{2} P^{2}=P_{p q}^{2} f(p)=R^{2} g(p)$, it follows that $P_{p q}$ is divisible by $R$. In conclusion, $\pm P_{p q}$ is characterized as such an $R$ of maximal degree.
Remark 5.2. Our proof of Theorem 5.1 is in a sense dual to Masuda's [6, §3]. In our notation, Masuda starts by essentially characterizing the Thom classes $\xi_{i}$ (see Remark 3.2) corresponding to invariant divisors as the non-zero elements of $H_{T}^{2}(\mathcal{G})$ with minimal support. He then reconstructs the fan $\Sigma$ by checking which products among the $\xi_{i}$ 's are non-zero. In the absence of a fan inducing the graph $\mathcal{G}$, we instead look at elements of $H_{T}^{*}(\mathcal{G})$ supported at single vertices, which correspond to fixed points.

Acknowledgments. The authors are grateful to the referee for several useful suggestions which have improved the presentation of the paper. M. F. was supported by an NSERC Discovery Grant. H. Y. was supported by JSPS Grant-in-Aid for Early-Career Scientists 19K14537, the bilateral program "Topology and geometry of torus actions, cohomological rigidity, and hyperbolic manifolds" between JSPS and RFBR, and Osaka City University Advanced Mathematical Institute (MEXT Joint Usage/Research Center on Mathematics and Theoretical Physics).

\section{References}

[ 1 ] M. Franz and V. Puppe, Exact cohomology sequences with integral coefficients for torus actions, Transform. Groups 12 (2007), no. 1, 65-76.

[ 2 ] M. Franz and V. Puppe, Exact sequences for equivariantly formal spaces, C. R. Math. Acad. Sci. Soc. R. Can. 33 (2011), no. 1, 1-10.

[ 3 ] M. Goresky, R. Kottwitz and R. MacPherson, Equivariant cohomology, Koszul duality, and the localization theorem, Invent. Math. 131 (1998), no. 1, 25-83.

[ 4 ] V. Guillemin and C. Zara, Equivariant de Rham theory and graphs, Asian J. Math. 3 (1999), no. $1,49-76$.

[ 5 ] H. Maeda, M. Masuda and T. Panov, Torus graphs and simplicial posets, Adv. Math. 212 (2007), no. 2, 458-483.

[ 6 ] M. Masuda, Equivariant cohomology distinguishes toric manifolds, Adv. Math. 218 (2008), no. 6, 2005-2012.

[ 7 ] M. Masuda and T. Panov, On the cohomology of torus manifolds, Osaka J. Math. 43 (2006), no. $3,711-746$. 